\title{
CONGENITAL POUCH COLON
}

\author{
KANIZHASINA
}

\section{Introduction:}

Congenital pouch colon is a condition associated with anorectal agenesis, and is defined as an anomaly in which whole or part of the colon is replaced by a pouch-like dilatation, which communicates distally with the urogenital tract by a large fistula. ${ }^{1}$ This condition has been seen as a curiosity ever since its first description in 1912 by Spriggs in a London Hospital Museum specimen with absence of the left half of colon and rectum. ${ }^{2}$ Various terms have been used to describe this entity including pouch-like dilatation of shortened colon, exstrophiasplanchnica, absence of colon and rectum, cystic dilatation of colon, short colon, colonic reservoir, congenital atresia of anus with short colon malformation, pouch colon syndrome, association of imperforate anus with short colon (AIASC) and congenital pouch colon. ${ }^{4-12}$ The term has now been included as rare variant, in the Krickenbeck classification of anorectal-malformations (ARM). ${ }^{13,14}$

Congenital pouch colon is seen much more frequently in India and neighbouring nations like Pakistan, Bangladesh and Nepal. Only a few reports have originated from China, Japan, Sweden, United Kingdom and other parts of the world. ${ }^{15}$ Majority of the patients present in the early neonatal period between 1 to 7 days. Occasionally if the fistula is large, especially in a female child the presentation may be late. There is a male preponderance in patients of congenital pouch colon. The sex ratio is 1.27:1. The incidence of this condition among all cases of ARM has been reported to be between 2.58 to $9 \% .{ }^{16}$

\section{Classification:}

The most widely accepted classification was given by Narsimha Rao et al ${ }^{10}$.

Correspondence to : Dr. Kaniz Hasina, Associate Professor, Department of Pediatric Surgery, Dhaka Medical College, Dhaka.

E-mail:kanizhasina@gmail.com
Type I: Normal colon is absent and the ileum opens directly into the colonic pouch.

Type II: The ileum opens into a short segment of caecum which then opens into the pouch.

Type III: Presence of a significant length of normal colon between the ileum and the colonic pouch.

Type IV: Presence of near normal colon with only the terminal portion of colon (sigmoid and rectum) converted into a pouch.

Type I and Type II are more common and account for more than $90 \%$ of cases.

Presently Wakhlu et al have attempted to further simplify the classification, based on the length of normal colon and management planning in relation to need of coloplasty. ${ }^{18}$

Type A (Partial short colon): Length of normal colon proximal to pouch is $>8 \mathrm{~cm}$.

Type B ((Complete short colon): Either absent normal colon or length of colon proximal to pouch is $<8 \mathrm{~cm}$.

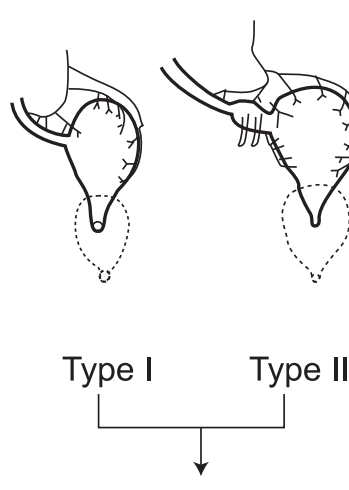

Complete
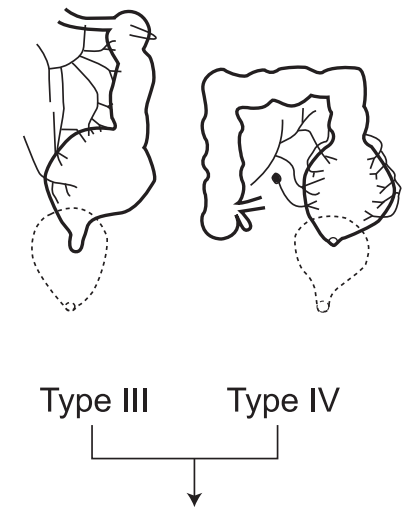

Incomplete
Fig.-1 


\section{Embryogenesis:}

1. Exactly not known.

2. Various workers have proposed different hypothesis. ${ }^{16}$

3. Vascular ischemia is the basis for occurrence of ARM. ${ }^{19}$

4. Vascular compromise in the region of hindgut (inferior mesenteric artery) during early intrauterine development causes abnormal, imperfectly developed shortened hindgut leading to pouch like dilatation of the colon by accumulation of meconium. ${ }^{19}$

5. The shortened aborted hindgut is unable to herniate into extracoelomic space and later, because of its extremely short length,cannot undergo normal rotation. ${ }^{19}$

\section{Anatomical Features:}

The mesentery of the pouch of variable size $(5-15 \mathrm{~cm}$ in diameter) is short and poorly developed, wall is very thick, the taenia coli are absent or ill defined, haustration and the appendices epiploicae are absent. The blood supply to the pouch is abnormal. The main pouch is supplied by the branches arising from the superior mesenteric artery that form a leash of vessels around it. There is no transitional zone between the pouch colon and the normal bowel. The pattern changes suddenly and sharply. The colon wall is thick and muscular with hypertrophied mucosa. Pelvic musculature is well developed in cases of short colon. The histology is also different and marked with two striking features; a very thick sub-mucosal layer and a criss-cross pattern of decussating fibres in the muscle coat. The normal longitudinal and circular pattern is found lost.

\section{Associated Anomalies:}

I. Genitourinary system

$\S$ Hydronephrosis

$\S$ VUR

$\S$ Posterior urethral diverticulum

$\S$ Renal aplasia and dysplasia

$\S$ Hypospadias

$\S$ Cryptorchidism

$\S$ Bicornuate uterus

$\S$ Double uterus/vagina

$\S$ Septate vagina

II. Gastrointestinal system

$\S$ Double appendix

$\S$ Absent appendix

$\S$ TEF and Esophageal atresia

$\S$ Malrotation
$\S$ Ileal atresia

$\S$ Duplication of gut

$\S$ Meckel'sdiverticuluma

III. Other anomalies

$\S$ Sacral agenesis

$\S$ Hemivertebrae

$\S$ Congenital heart disease

$\S$ TEV

$\S$ Prune belly syndrome

Clinical Presentation:

1. Early Presentation-

- Present in early neonatal period between 1 to 7 days

- Gross abdominal distension

- Bilious vomiting

- ARM

- Meconiumuria

- Pneumaturia

- Persistent cloaca in female

2. Late Presentation-

$\S$ When fistulous connection with urogenital tract is large, the child may present quiet late even few months after birth.

$\S$ At that time, the child may be undernourished, constipated and passing faeces from an abnormal opening.

\section{Investigations:}

1. Prone cross-table lateral X-ray- a large bowel loop with single air fluid level occupying more than $50 \%$ of the abdominal cavity in a patient with anorectal malformation is a diagnostic sign of congenital pouch colon.

2. Other investigations-

$\S \quad$ To assess the general condition of the baby

$\S$ To detect associated anomalies

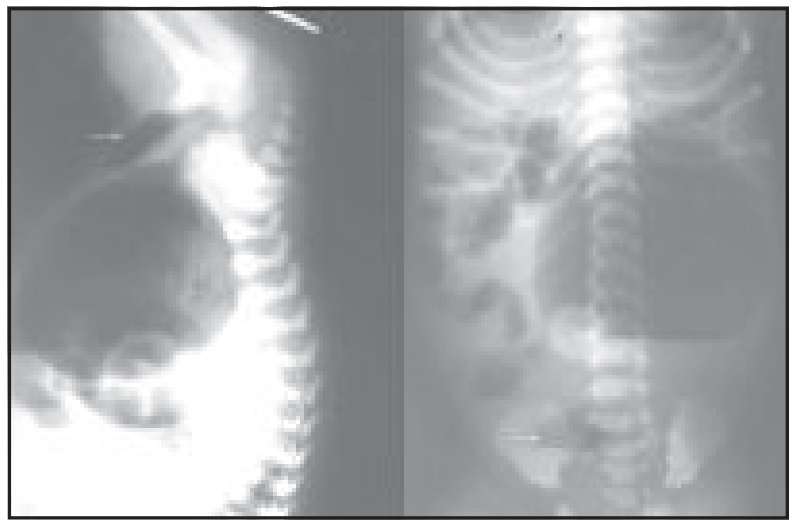

Fig. - 2: 
Preoperative Staging: (For prognostication) ${ }^{16}$

\begin{tabular}{lccccc}
\hline Group & Weight & Time of Presentation & Sepsis & Complication & Mortality\& Prognosis \\
\hline I & $>2.5 \mathrm{~kg}$ & $<24$ hours & - & - & $0 \%$, excellent \\
II & $2-2.5 \mathrm{~kg}$ & $<48$ hours & + & - & $20 \%$ \\
III & $<2 \mathrm{~kg}$ & $>48$ hours & + & + & $60 \%$ \\
\hline
\end{tabular}

Management:

1. Preoperative Preparation-

$\S$ Nothing per oral

$\S$ Nasogastric suction

$\S$ Correction of dehydration and electrolyte imbalance

$\S$ Antibiotics

$\S$ Injection of Vitamin K

$\S$ Maintenance of body temperature

$\S$ Catheter in situ to measure urine output and to decompress bladder

2. Surgical Treatment-As the dilated pouch is always abnormal in anatomy and function; surgical procedures should ideally include an excision of the dilated pouch with an end colostomy at birth and a definitive pull through later.

$\S$ Single Stage-A single stage pullthrough can be undertaken in newborn stage in the incomplete type of the pouch colon, only if the condition of the baby permits.

$\S$ Staged Surgery-Diversion, definitive surgery and restoration of gut are the ideal steps in complete pouch colon.

- Window colostomy/proximal ileostomy/end colostomy (after division of fistula)/end colostomy after coloplasty

- Abdomino-PSARP

- Stoma closure

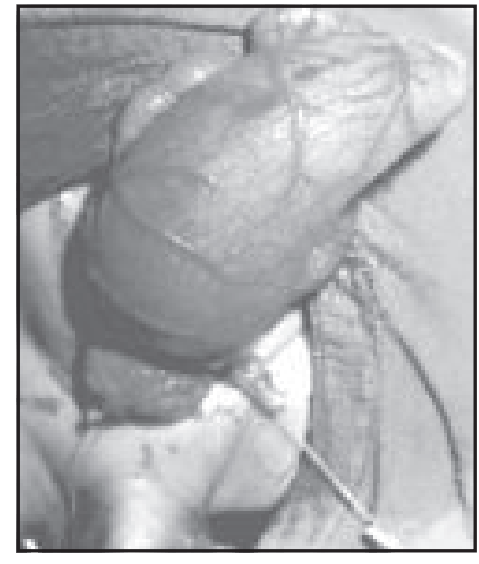

Fig.-3
A coloplasty procedure would be required to retain about $15 \mathrm{~cm}$ length of pouch colon in the form of a tube, to be brought out as an end colostomy. A window colostomy from the body of the pouch, though very easy and quick to perform in emergency, should be discouraged as it results with frequent prolapses, stasis and fecoloma formations. Also, a pull through procedure at the time of performing coloplasty should not be preferred in the newborn stage as it is associated with high morbidity and mortality. Any attempt to make a coloplasty longer than $15 \mathrm{~cm}$, not only results in stasis and dilation of the segment but also causes frequent complications in the post pull through period.

\section{Postoperative Complications:}

The complications associated with ileostomy are -

1. Excoriation

2. Bleeding

3. Electrolyte imbalance

4. Malnutrition

5. Dehydration

6. Vitamin deficiency

The complications associated with window colostomy are-

1. Prolapse

2. Stenosis

3. Fecal retention

4. Urinary tract infection

5. Pouch dilatation

6. Pouchitis

7. Enterocolitis

8. Persistent abdominal distension

9. Massive mucosal prolapse

10. Para-colostomy hernia and

11. Evisceration.

The complications associated with coloplasty are-

1. Suture line leak

2. Wound dehiscence

3. Colonic dilatation

Common complications of any anorectal reconstruction-

1. Anal stenosis

2. Mucosal prolapse

3. Encopresis 
4. Chronic dilatation of retained pouch

5. Chronic fecal retention

6. Retention with overflow and

7. Fecal soiling

Boys with Congenital Pouch Colon often have a wide fistula with urinary tract. Metabolic acidosis and failure to thrive is also seen secondary to reflux of urine in bowel and absorption. ${ }^{3}$

Postoperative Care:

I. Short term postoperative care-

1. Taking care of stoma/perineal excoriations

2. Keeping the stool soft and

3. Preventing stenosis of neo-anus with regular dilatation program

II. Long term post-operative care-

1. Bowel training

2. Stool softeners

3. Diet modification

4. Monitoring of the urinary tract for early detection and management of neurogenic bladder, VUR and other urinary problems.

\section{Follow-up:}

The follow-up examination is done initially after 15 days and then after 1 month. Subsequently patient is called every 3 months for 1 year and six months thereafter. Anal dilatation is started after 3 weeks of surgery and continued till required. ${ }^{16}$

\section{Conclusion:}

As the awareness about congenital pouch colon has grown and the successful management has become possible, this condition is being found in increasing frequency to be associated with ARM.Though the overall mortality of congenital pouch colon continues to be high, ranges between $30 \%$ to $50 \%$,once the baby survives and undergoes all stages the results in terms of continence, physical, motor and behavioural development are usually satisfactory.

\section{References:}

1. Gupta DK, Sharma S. Congenital Pouch Colon, in Anorectal Malformations, 1st ed. Hutson J, Holschneider A, editors. Springer Heidelberg: 2006. p. 211-22.

2. Spriggs NJ. Congenital occlusion of the gastrointestinal tract. Guys Hosp Rep 1912; 766: 143.

3. Gupta DK, Sharma S. Congenital pouch colon Then and now. J Indian AssocPediatr Surg. 2007; 12: 5-12.
4. Trusler GA, Mestel AL, Stephens CA. Colon malformation with imperforate anus. Surgery 1959;45:328-34.

5. Spencer R. Exstrophiasplanchnica (exstrophy of the cloaca). Surgery 1965;57:751-66.

6. BluntA, Rich GF. Congenital absence of the colon and rectum. Am J Dis Child 1967;114:405-6.

7. Shafie M. Congenital short intestine and cystic dilatation of the colon associated with ectopic anus. J PediatrSurg 1971;6:76.

8. Singh S, Pathak IC. Short colon associated with imperforate anus. Surgery 1972;71:781-6.

9. Gopal G. Congenital rectovaginal fistula with colonic reservoir. Indian J Surg 1978;40:446.

10. NarsimhaRao KL, Yadav K, Mitra SK, Pathak IG. Congenital short colon with imperforate anus (pouch colon syndrome). Ann PediatrSurg 1984;1:159.

11. Wu YJ, Du R, Zhang GE, Bi ZG. Association of imperforate anus with short colon: A report of eight cases. J PediatrSurg 1990;25:282-4.

12. Chadha R, Bagga D, Gupta S, Prasad A. Congenital pouch colon: Massive redilatation of the tubularized colonic pouch after pull-through surgery. J PediatrSurg 2002;37:1376-9.

13. Gupta DK. Anorectal malformations-Wingspread to Krickenbeck. J Indian AssocPedSurg 2005;10:79.

14. Holschneider A, Hutson J, Pena A, Beket E, Chatterjee S, Coran A, et al. Preliminary report on the International Conference for the Development of Standards for the Treatment of Anorectal Malformations. J PediatrSurg 2005;10:1521-6.

15. Gupta DK. Congenital pouch colon: Present lacunae. J Indian AssocPediatrSurg 2007;12:1-2.

16. Wakhlu AK, Pandey A; Congenital Pouch Colon; Text book of Neonatal Surgery;Edited by D.K.Gupta; $1^{\text {st }}$ edition; 2000; 240-248.

17. Wakhlu AK, Pandey A, Wakhlu A et al; Coloplasty for Congenital Short Colon; J of PediatrSurg; 1996; 31:344-348.

18. Wakhlu AK, Wakhlu A, Pandey A et al; Congenital Short Colon; World J of Surg; 1996; 20: 107-114.

19. Chadha R, Bagga D, Malhotra CJ et al. The Embryology and Management of Congenital pouch colon associated with Anorectal Agenesis. J Pediatr Surg 1994; 29(3): 439-446. 